\title{
Dynamics of Blockchain Implementation - A Case Study from the Energy Sector
}

\author{
Simon Albrecht \\ University of Freiburg \\ simon.albrecht@is.uni-freiburg.de \\ Jens Strüker \\ Fresenius University \\ jens.strueker@hs-fresenius.de
}

\author{
Stefan Reichert \\ University of Freiburg \\ reichert@iig.uni-freiburg.de \\ Dirk Neumann \\ University of Freiburg \\ dirk.neumann@is.uni-freiburg.de
}

\author{
Jan Schmid \\ Fresenius University \\ jan.schmid@,hs-fresenius.de \\ Gilbert Fridgen \\ University of Bayreuth \\ gilbert.fridgen@uni-bayreuth.de
}

\begin{abstract}
This case study analyzes the impact of theory-based factors on the implementation of different blockchain technologies in use cases from the energy sector. We construct an integrated research model based on the Diffusion of Innovations theory, institutional economics and the Technology-Organization-Environment framework. Using qualitative data from in-depth interviews, we link constructs to theory and assess their impact on each use case. Doing so we can depict the dynamic relations between different blockchain technologies and the energy sector. The study provides insights for decision makers in electric utilities, and government administrations.
\end{abstract}

\section{Introduction}

Blockchain is making headlines as the next step in the evolution of the internet in contemporary tech, finance and energy press outlets. Simultaneously, the emergence of renewable, Distributed Energy Resources (DER) and smart grids creates a network, in which innumerable devices can automatically communicate with each other. Regardless of the hype, the potential for blockchain in the energy sector appears promising. The automation of processes, disintermediation and the rethinking of value chains grant degrees of freedom for innovative applications and business models. Yet, there are only few market-ready blockchain services, but rather pilot projects in which specific applications are tested. Although most publications refer to 'the' blockchain, a comprehensive view requires a differentiation between multiple blockchain technologies. Therefore, we analyze the distinctive characteristics of these technologies and the socioeconomical and institutional context and their relationship to one another. To explain the dynamics, we address the following research question:

How and by which factors is the implementation of blockchain technologies in energy sector use cases affected?

We utilize a theoretical framework and in-depth interviews for this analysis. To our knowledge, this is the first paper to evaluate the implementation of different blockchains in use cases from the energy sector. Former research has aimed to define a decision model for blockchain adoption based on the TOE framework [8]. However, these authors do not make the crucial differentiation between distinct types of blockchain technologies and different business areas.

The paper is structured as follows: Section 2 describes the research process, followed by an overview of seminal theoretical literature in section 3 . Subsequently, section 4 provides essential background information for the energy sector and blockchain technologies. In section 5, relevant factors impacting on decision makers are discussed, while section 6 analyzes implications of these factors for energy use cases. The concluding section 7 summarizes the most important insights and limitations and provides an outlook on future research.

\section{Methodology}

While there is a great body of non-scientific literature on the application of blockchains in different use cases [9, 20, 27], few articles are published in peerreviewed scientific journals. After an initial literature search with literature databases (EBSCOhost, Google Scholar) we found Yli-Huumo's [49] overview as an 
identifier for research gaps. Based on that we formulated preliminary research questions and initiated the search for a suitable framework. After reviewing the seminal body of literature in this field, we decided to use an integrated framework based on the theoretical background described in Section 3,

While searching for appropriate interviewees, we intentionally used our professional network instead of random sampling to consult experts in specific domains of blockchains and energy. We found 22 appropriate interviewees from three distinct groups: (A) Energy sector decision makers (B) blockchain experts (C) researchers. We designed a pool of a dozen open interview questions based on our research aims and adjusted them according to individual interviewees' parameters (experience, attitude, position, etc.). All interviews were executed on the telephone, except for two email interviews. We used an in-depth and semistructured interview design as suggested by Bewley [3]. Subsequently, we used open coding to identify relevant variables in the qualitative data, and axial coding to connect these variables to general factors [13]. We then assigned these general factors from our data to the framework (Figure 1).

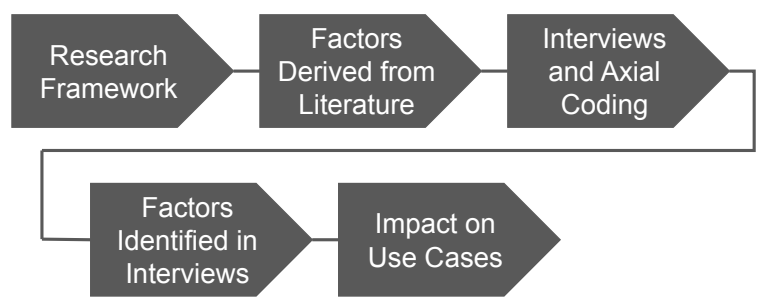

Figure 1. Research process

To design a valid case study, we used multiple sources of evidence in terms of different mediums (phone, email and literature) as well as different stakeholders at different points of the research. To safeguard reliability as suggested in [48], we repeated the coding process by different researchers [29]. We maintained a database with all data about the interviews, as well as a protocol to document the ongoing progress of the study for all participating researchers.

\section{Theoretical framework}

We reviewed the seminal literature for technological innovation while searching for an appropriate framework four our study. Regarding the domain of blockchains, our analysis requires a sound theoretical foundation that reflects (a) the specific properties of the blockchain technology, (b) the dynamics of organizations utilizing blockchains, (c) the highly regulated nature of the energy sector, and (d) the external effects of such a networked technology.

During this process, we reviewed the Technology Acceptance Model (TAM) [42] and the Unified Theory of Acceptance and Use of Technology (UTAUT) [43], but we eventually dismissed these frameworks due to their focus on individual behavior and lacking importance of environmental and institutional factors. In contrast to that, the commonly applied Diffusion of Innovations theory (DOI) investigates underlying mechanisms of diffusion and assesses how an innovation can be implemented in a social system. The most prominent contribution in this field was made by Rogers [34], who discusses the dynamics between the technological innovation itself, organizations to adopt the innovation and communication channels of the regarded social system, which addresses (a) and (b).

Building on this, DePietro et al. [16] established the Technology-Organization-Environment framework (TOE) to display constraints and opportunities of a technological innovation in organizations. They complement Rogers' work by adding an environmental component to the framework. This component flexibly embodies relations of the organization towards external units such as government, competition, or technology infrastructure, addressing (c).

DOI has been criticized for disregarding standards, network externalities and path dependence [25]. Accordingly, DOI researchers are advised to consider the nature of the technology, the critical role of institutional structures, strategies of stakeholders and the installed infrastructure. Similarly, critics of TOE state, that the flexibility of the framework is gained at the expense of accuracy. To address this, the incorporation of network externalities, described for instance in [37] has been suggested by [1]. In order to supplement the shortcomings of DOI and TOE as well as to address (d), we took on an economic perspective on blockchains as suggested by [15], drawing on seminal works in the field of institutional and information economics such as [6] and [37], considering network externalities and rent-seeking behavior.

Based on the reviewed literature, we decided to construct a flexible integrated research framework to carry out a phronetic analysis as proposed in [17]. Phronetic research is a pragmatic interpretation of the research object and thus well applicable to case studies. It does not aim to develop new theories or yield causal evidence, but rather to contribute to practical rationality by investigating the dynamics of power and relations in a social context. With this approach, we aim to reflect the dynamics of blockchain in the energy sector. 


\section{Background}

In this section, we differentiate the characteristics of blockchain technologies and we illustrate contemporary developments in the environment of the energy sector.

\subsection{The energy sector}

Energy markets are facing changes induced by technological and socio-economic developments. The energy generation transitions from conventional thermal power plants to distributed energy resources. This induces fluctuating supply, increasing uncertainty. Energy trade becomes more complex. Additionally, smart meter gateway tethered devices are replacing analogous meters. The heterogeneous generation is causing high volatility. Accordingly, supply and price are subject to high levels of uncertainty. DER such as power-heat coupling plants, photovoltaic installations and biogas plants are becoming more popular for residents and local organizers. An increasing share of energy demand can be provided locally, making the grid balancing a challenging task. The changes of the energy market increase participation and empowerment for customers, enabling them to optimize domestic consumption and to switch retailers [14].

\subsection{Distributed ledger technology}

A decade ago, the Bitcoin white paper published by Satoshi Nakamoto [28], introduced the revolutionary concept of blockchains. The so-called distributed ledgers are records of transactions distributed to every computer in the participating network [40]. Unlike normal databases, it is updated by all network participants. This process is called Proof-of-Work (PoW). Miners compete against each other to solve an algorithmic puzzle and the fastest contestant updates the database. Trust, which is usually provided by intermediaries is achieved by the collaboration of the masses [41]. While every entity that participates in a blockchain can review the stored information, changes to the database can only be implemented by reaching a consensus. Contemporary blockchains have started to include so-called smart contracts that are negotiated between parties, stored in code and executed autonomously. Smart contracts enhance the blockchain technology to a system of distributed applications and markets [30]. While the term 'blockchain' often refers to permissionless distributed ledgers, several publications differentiate between those, permissioned, and consortium blockchains $[2,44]$.
Table 1. Blockchain characteristics $[2,44]$

\begin{tabular}{|c|c|c|c|}
\hline & Public & Private & Consortium \\
\hline Access & Permissionless & Permissioned & Shared Perm. \\
\hline $\begin{array}{l}\text { Personal } \\
\text { information }\end{array}$ & Pseudonymity & Known & Known \\
\hline $\begin{array}{l}\text { Device } \\
\text { authentication }\end{array}$ & Not required & Required & Conditional \\
\hline $\begin{array}{l}\text { Consensus } \\
\text { mechanism }\end{array}$ & PoW, PoS & $\begin{array}{l}\text { PoS, PoA, } \\
\text { PBFT }\end{array}$ & $\begin{array}{l}\text { PoW,PoS, } \\
\text { PoA }\end{array}$ \\
\hline Security & $\begin{array}{l}\text { Decentralized } \\
\text { control }\end{array}$ & $\begin{array}{l}\text { Single point } \\
\text { of failure }\end{array}$ & Various \\
\hline $\begin{array}{l}\text { Transaction } \\
\text { speed }\end{array}$ & Low (PoW) & Rather High & $\begin{array}{l}\text { Higher than } \\
\text { public }\end{array}$ \\
\hline $\begin{array}{l}\text { Energy } \\
\text { consumption }\end{array}$ & High (PoW & Rather Low & Rather low \\
\hline System costs & High & Rather low & Medium to low \\
\hline $\begin{array}{l}\text { Individual } \\
\text { costs }\end{array}$ & Low & Rather high & Various \\
\hline
\end{tabular}

\subsection{Public (permissionless) blockchains}

The prominent blockchains Bitcoin and Ethereum are permissionless and public (Table 1). These ledgers are accessible to anyone and participants are represented by a random ID (pseudonymity). There is no central provider to supervise the ongoing traffic or to admit new applications. Permissionless blockchains allow the participation of random unknown devices without initial checks of trustworthiness. These blockchains historically rely on the PoW consensus mechanism, in which every miner can validate new data blocks. However, some blockchains have already implemented efficient alternatives, such as the "Proof-of-Stake" mechanism (PoS), which randomly selects network participants who own a stake of the network's tokens to validate transactions [7].

\subsection{Private (permissioned) blockchains}

Permissioned (private) blockchains only grant access to known participants, who might have rights to read and/or write data. The system provider has full control over the blockchain and he knows all participants a priori. Generally, he can roll back certain processes making permissioned blockchains potentially reversible. The validation of single blocks is possible at much lower resource consumption and higher speed, as not all participants are simultaneously working on the solution of the algorithmic puzzle. Instead, in the Proofof-Authority (PoA) consensus mechanism, a single node generates new data blocks. The formerly described $\mathrm{PoS}$ can also be employed in permissioned 
environments. For instance the open-source PoS engine Tendermint can be used as the base infrastructure for different types of blockchains [7]. A similar approach, the Practical Byzantine Fault-Tolerant (PBFT) consensus algorithm [10] is utilized by Hyperledger Fabric [18]. Based on PBFT, a single authority issues permissions for membership to a network of known participants, in which only few whitelisted nodes validate transactions.

\subsection{Consortium blockchains and multi-purpose technologies}

Consortium blockchains are a compromise between public and private blockchains in which only verified participants can validate blocks. These blockchains offer the possibility to be tailored towards the specific requirements of the consortium, e.g. by removing pseudonymity. Apart from the three blockchains categories established in prior works [44], there is a multitude of protocols, forks and platforms that can be incorporated or built on as blockchain components. For instance, Tendermint is most prominently used as the core of the Cosmos project, which aims at enabling a seamless exchange of tokens between different blockchains [7, 23]. Similarly, Ethereum's co-founder Gavin Wood developed Polkadot [47], a relay channel network to parallelize different chains. The other Ethereum co-founder Vitalik Buterin is involved in Plasma, a concept to scale different blockchains in Ethereum smart contracts [32]. Due to the appearance of the versatile technologies stated above, the categorical differentiation of distributed ledger technologies has become more complex throughout the last year. For this work, the main characteristic to categorize a blockchain is the access to the network and consensus mechanism that defines which entities are permitted to validate transactions.

\section{Critical factors in the domain of blockchain technologies}

In this section, we assess the innovation diffusion factors from the theory, linked with the most relevant factors impacting on the use of blockchain technologies from our qualitative data. The mapping of axial codes from the interviews to theory-based factors is depicted in Table 2.

\subsection{Market power and competitive pressure}

Competitive pressure is an established pillar of innovation diffusion research. Customer power, threat of new entrants, industry rivalry and threat of substitutes impact on market power dynamics [33]. Incumbents holding significant market power, generally aim to keep the status quo instead of sponsoring innovative technologies. If an innovation arises, pressure from startups and customers increases. To secure the market share, incumbents will likely engage with the innovation, yet prevent the occurrence of network externalities that may benefit competitors. Therefore, a company in a dominant market position may not support efforts to foster interoperability. Instead, it may capture this process, tighten path dependencies and aim to raise switching-costs [37].

\subsection{Regulation and rent-seeking behavior}

The regulatory environment refers to the support or barriers given by a government authority and is considered to be a major factor for technological innovation [50].

Although no blockchain-specific regulation has passed legislation so far, obligatory guidelines for the registration as market participants may be a an obstacle for permissionless blockchains [36]. Further, regulation generally relies on the written form. Conventional contracts are characterized by a certain degree of openness, can be interpreted in certain ways and are not suitable to be represented by a smart contract. When a contracted service is not being delivered, no mediator will enforce laws and nobody may be found liable in a permissionless blockchain [2]. The application of smart contracts is therefore less problematic within permissioned blockchains, where all participants are known.

Since regulation is part of a complex legislative process involving government agents and public representatives, there is potential for political rentseeking as described in [6]. Blockchain technologies generally pose a threat for power-holding entities as they efficiently distribute information and decrease the necessity for established stakeholders.

\subsection{Technology characteristics}

The emphasis on technology characteristics in the DOI theory equates with the categorization of technology in the TOE framework [1]. The interviews exhibited three codes linked to technology characteristics.

Transparency. Lack of trust forces trading parties either to rely on manual processes or to engage presumably trustworthy intermediates. Whenever such an intermediary agent is employed, principal-agent problems and rent-seeking behavior induced by incomplete contracts can arise [11]. These manifestations may be addressed with distributed 
ledgers. In a permissionless blockchain all current and historic transactions are publicly viewable, creating full transparency of the network. Doing so, permissionless blockchains can eliminate opportunism [15]. In contrast, permissioned blockchains can be configured to provide transparent information to network participants (or even the public). However, the governing authority can easily withdraw this feature. Accordingly, optional transparency in the absence of real immutability may not foster trust in the same way a permissionless blockchain can. Incomplete contracts remain an issue since smart contracts in blockchain are less complete than conventional ones [2]. Unspecified when-ifrelations in smart contracts, can still be exploited by human behavior. Accordingly a full substitution of trust by blockchains is only feasible in markets using nonhuman agents like a Decentralized Autonomous Organization (DAO), while human involvement leaves room for the execution of power dynamics [15].

Transaction speed. Today the capacity of permissionless blockchains is sufficient for discrete tasks. For a large-scale use of the technology however, the transaction speed of is one of the key limiting factors. The Ethereum blockchain currently allows only for 10-20 transaction per second (tps). For comparison, the Visa network carries out 1667 tps (PayPal 193 tps) on average [51]. Current permissionless blockchains are not yet suitable for high-frequency transactions when PoW is used. In contrast to that, permissioned blockchains do not have any technology-specific restrictions regarding the transaction speed. All nodes in the network are known and trustworthy, thus the validation of transactions is trivial. The PBFT mechanism is built to reach consensus fast and efficiently, allowing tens of thousands tps [45]. Currently, the Energy Web Foundation (EWF), funded by the Rocky Mountain Institute is establishing a PoAbased permissioned blockchain platform. Building on Ethereum as a basis, this platform uses so-called state channels, in which results of transactions are grouped together in a smart contract. This significantly increase the scalability of blockchain transactions. The state channel network Kovan for instance reaches 1.000 tps [52].

Transaction costs. Generally, the innovation literature considers costs to be an inhibitor of technology use [24]. The experts from our interviews considered this true for permissionless blockchains. At the same time, they identified permissioned blockchains' cost-effectiveness to be a major advantage. Permissionless blockchains' system costs (with PoW) are perceived as a key obstacle. The operation costs of the Ethereum network amounts $93.440 .000 \$$ per year [39]. However, these operational costs mostly consist of the transaction fees. A simple Ethereum transaction costs about $\$ 0,12$ cents on the current exchange rate [46], which is still inexpensive compared to existing payment service providers (PayPal: $\$ 0.30$ plus $2.9 \%$ of the volume [53]).

\subsection{Security}

In the domain of security, our qualitative data exhibited codes that equated to the basic components of computer security (confidentiality, integrity and availability [4]).

Table 2. Axial coding from qualitative data

\begin{tabular}{|c|c|c|c|}
\hline Theory-based factors & Axial codes & $\mathbf{n}$ & Exemplary quote from interviews \\
\hline Competitive Pressure & Market power & 17 & $\begin{array}{l}\text { Customers are longing for someone who is in charge, a service } \\
\text { contact. This only works in permissioned blockchains. }\end{array}$ \\
\hline $\begin{array}{l}\text { Regulatory Environment } \\
\text { Rent-Seeking Behavior }\end{array}$ & Regulation & 14 & $\begin{array}{l}\text { The current regulation regarding grid fees prevent new business } \\
\text { models and thus innovation. }\end{array}$ \\
\hline \multirow{3}{*}{ Technology characteristics } & Transaction Speed & 12 & $\begin{array}{l}\text { The potential for permissionless blockchains is limited by } \\
\text { transactions speed. }\end{array}$ \\
\hline & Transparency & 16 & $\begin{array}{l}\text { When all generation data is transparent, we will experience less } \\
\text { conflicts regarding shared ownership of pv systems. }\end{array}$ \\
\hline & Transact. Costs & 20 & In some areas, we can reduce about $80 \%$ of the operational costs. \\
\hline \multirow{3}{*}{ Security } & Integrity & 18 & $\begin{array}{l}\text { Inside the blockchain all data is safe. It is the interface to the } \\
\text { physical world where threats occur. }\end{array}$ \\
\hline & Confidentiality & 12 & The right to be forgotten is not applicable in blockchains. \\
\hline & Availability & 4 & $\begin{array}{l}\text { Permissionless blockchains are always available. Not even the IT } \\
\text { systems of stock exchanges can provide that. }\end{array}$ \\
\hline \multicolumn{4}{|l|}{ Compatibility } \\
\hline $\begin{array}{l}\text { Network Externalities } \\
\text { Path Dependencies }\end{array}$ & Interoperability & 14 & $\begin{array}{l}\text { In permissionless blockchains, devices that have not met before } \\
\text { can spontaneously make transactions with each other. }\end{array}$ \\
\hline
\end{tabular}


Integrity. The immutability feature of blockchains (the prevention of unauthorized change) is a key characteristic for data integrity. A permissionless blockchain is immutable, as it is almost impossible to alter stored information without being detected. Doing so, an attacker would need $50 \%$ of the network's computing power when based on PoW [35]. Although the resilience against internal or external threats has yet to be investigated, a major vulnerability in a Decentralized Application (DApp) has been exposed by the prominent case of the so-called "Ethereum DAOHack", in which an incomplete smart contract enabled a financial exploit.

Due to the similarity to centralized systems, permissioned and consortium blockchains do not exhibit a specific advantage regarding integrity, since nodes in permissioned blockchains can easily agree to alter the existing database.

Availability. In the TOE framework, availability refers to the accessibility of a given technology for an organization. Unlike in centralized architectures, blockchains are not subject to a single point of failure. Although an attack may hit the permission-issuing entity in private blockchains, several other nodes store identical copies of the database, safeguarding its availability. Even further, permissionless blockchains are shared among large numbers of participants [41]. If one entity of the network breaks off, the remaining participants will keep the system running. However, while a distributed infrastructure is resilient to shocks, most DApps run on few conventional web servers, which can be hacked.

Confidentiality. Although permissionless blockchains provide pseudonymity, it is possible to identify entities behind the unique address by analyzing the data on blockchain [38]. Blockchains hold personal information that remain in the distributed database due to the immutability characteristic. Although the legislative environment of many countries grants customers the right to have personal information removed or transferred (data portability), this is not feasible in a permissionless blockchain unless a hard fork of the entire chain is carried out. Although this affects permissioned blockchains as well, central authority makes it easier to cut-off parts of the transaction history.

\subsection{Compatibility and network externalities}

In the DOI literature, compatibility refers to the suitability of a technology within a given technological system of an organization [34]. Consistent with that, our interviewees identified the interoperability of different devices within networks as a major determinant for technology implementation. The value of a network is determined by the number of its participants [5]. When path dependence is present, the establishment of a practical migration strategy is important to secure a critical mass of network traffic. One way is to cooperate with other stakeholders and engage in standardization efforts to minimize switching costs [37]. In this context, the International Organization for Standardization has established a technical committee (ISO/TC 307) for interoperability and data exchange on distributed ledger technologies [22]. Regarding the realization of network effects, permissionless blockchains are advantageous by allowing spontaneous authentication of devices. Alternatively, hybrid- and multi-purpose chains able to combine interoperability and process efficiency.

\section{Impact on energy use cases}

After the identification of factors derived from literature and interviews, we now discuss their impact. Figure 2 depicts the line of argument between the identified factors and their relationship to use cases from the energy sector. Based on the qualitative data, relationships with strong effects are displayed in bold, while conditional or and weak relationships are displayed in dashed lines.

\subsection{Microgrids and local residential p2p-trade}

The prominent showcase built by LO3 and ConsenSys, the Brooklyn Microgrid (BMG) has proven technical feasibility of permissionless blockchains in microgrids. Similarly, the innogy spin-off company Conjoule operates a platform, allowing residential prosumers to sell excess electricity to local consumers, which is currently being piloted in two German cities [54]. The BMG use case's market design has been evaluated in [26]. The authors consider the regulatory environment to be major challenge for microgrids. Residential P2P-trade is illegal in most jurisdictions and only feasible in the BMG by incorporating $\mathrm{LO} 3$ as a licensed utility. While the BMG originally relied on the conventional Ethereum blockchain, LO3 has recently teamed up with Siemens to develop a permissioned Ethereum solution, incorporating the Tendermint consensus mechanism [26].

In a multi-directional cellular energy distribution system, there is a necessity for entities that ensure compliance with physical restrictions. Grid users must be known for that reason and possibly as well to fulfill other legal and regulatory requirements (taxation, know-your-customer laws). Accordingly, a private entity or consortium simplifies the operation of a microgrid. Further, the energy sector is experiencing a shift of power dynamics towards customers. 
Blockchains eliminate the need for intermediaries since transactions can be placed without third parties [40]. The increasing power of consumers incentives utilities to secure their market power. Customer-friendly products like smart home applications may be offered to influence switching costs, tighten path dependencies and thus prevent the realization of network effects. Simultaneously, cooperation with rent-seeking political representatives may occur to keep regulatory barriers in place, minimizing market threats of prosumer-driven projects.

\subsection{Grid services}

The increasing number of DER with fluctuating generation challenges existing means for grid monitoring and balancing. Based on a permissioned blockchain, Ponton deployed a software (Gridchain) in Austria to coordinate balancing power request between Transmission System Operators (TSOs), Distribution System Operators (DSOs) and aggregators within seconds [55]. A different project started as joint venture of transmission grid operator Tennet and the battery storage producer sonnen, aims to reduce the need for redispatch measures with home battery storage by utilizing the permissioned Hyperledger Fabric [56].
Also, the Rocky Mountain Institute is investigating blockchain based platforms applicability for DSOs [57]. Therefore, the associated Energy Web Foundation (EWF) is currently evaluating this use case for their permissioned blockchain solution.

Monitoring the status of the grid and taking stability measures requires high-integrity data, which is available constantly and in near-real-time for a small number of participants (DSO, TSO). Today, only permissioned projects are focusing these services, since the grid is considered a critical infrastructure and the regulatory environment will only grant a small number of entities insight and control.

\subsection{Wholesale electricity markets}

Blockchains enable direct electricity trade without intermediaries. The Ponton Enerchain tool currently enables the wholesale trade for two dozen European utilities by the use of the Tendermint consensus algorithm for permissioned blockchains [31]. In contrast to that, ConsenSys is currently initializing Grid+, a project aiming to use a permissionless Ethereum-based platform incorporating state channels to connect consumers directly to the wholesale market [12]. The utilization of a state channels network can safeguard

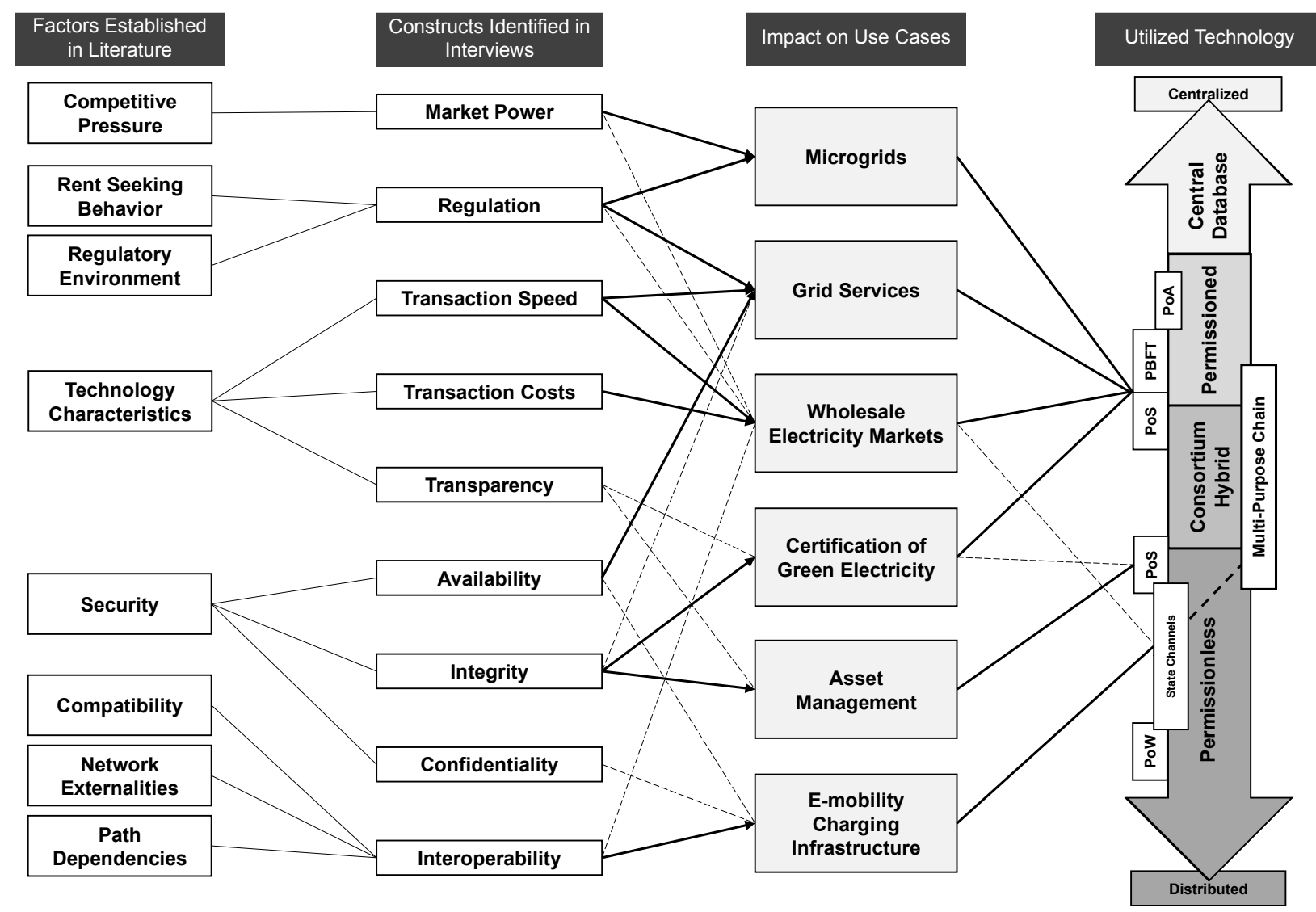

Figure 2. Research framework and relationships between factors and use cases 
transaction speed and scalability of a permissionless blockchain to realize network externalities.

While the reduction of transaction costs through disintermediation and transaction speed are major factors for wholesale trade, market power and rentseeking behavior have relevant impact, as stakeholders (traders and exchanges) may prefer consortiumcontrolled or permissioned blockchain solutions. A permissionless blockchain may only be favored when a critical mass of participants can be achieved by an appropriate scalability mechanism as used in multipurpose chains.

\subsection{Asset management}

The monitoring, metering and status documentation of specific energy resources can be coordinated via blockchains. This way, data regarding ownership structures, load profiles, and maintenance status can be made available for engineers and investors. Currently, one of the interviewees is initializing an asset management pilot study, utilizing the Ethereum blockchain for valuation of DER. Consulting services such as due diligence or asset valuations for municipal utilities can be automated with the aim to make small assets fungible. Investors of DER can benefit from transparent and high-integrity information. In contrast to grid services, in asset management a higher number of network participants may rely on the stored data. Therefore, employing a permissionless blockchain requires a scalable consensus mechanism like PoS.

\subsection{Certification of green electricity}

Renewable power plants typically must be certified by multiple institutions through costly processes to trade Renewable Energy Certificates (REC). Residential customers do usually not receive reliable information on their actual share of green energy. Therefore, the startup StromDAO has implemented a real-time electricity account, utilizing the a PoA version of the Ethereum blockchain. The electricity consumption of households is being evaluated by an index indicating the share of renewables in the regional energy mix. Depending on their consumption and procurement behavior, participating households receive tokens (Grünstromjetons) corresponding to their individual share of green electricity [19]. A different approach is tested by the Chinese Energy-Blockchain Lab using IBM's Hyperledger. This permissioned blockchain serves as a common ledger for carbon emission quotas to incentivize ecological behavior among China's industrial companies. Hyperledger allows transactions to be either confidential or public, fostering the transparency of the energy generation process [58].
A green energy certificates ledger must build on reliable information, accessible to stakeholders and citizens. While both permissionless and permissioned blockchains can grant transparency of information, the latter system can potentially disregard immutability and thus data integrity by cutting off the transaction history. When such a system is used to monitor ecological policies, decision makers may exhibit rent-seeking behavior when interest groups push for an installment of manipulable permissioned systems.

\subsection{E-mobility charging infrastructure}

The infrastructure of charging stations for electric vehicles is defined by a vast number of different providers with different procedures for billing and accounting. Currently, transactions are subject to numerous underlying processes between providers and intermediaries to identify users. The startup share \& charge, a spin-off founded within the innogy innovation hub, currently operates a blockchain charging infrastructure with more than 1200 stations, partly owned by private citizens [21]. The company is using smart contracts on top of the permissionless Ethereum blockchain, to grant automatized transactions between charging stations and electric vehicles.

Besides confidentiality of personal location data, and availability to safeguard charging at any given time, emobility requires a system in which unknown nodes can carry out spontaneous transactions without prior identification. Although this could be provided by a permissionless blockchain as executed by [21], the emobility environment already exhibits path dependence in form of pre-existing technologies. Further, devices have to be linked in order to enable compatibility in terms of seamless transactions and the exploitation of network externalities. Accordingly, the utilization of multi-purpose chains may be of interest for incumbent stakeholders. Alternatively, a permissionless blockchain that already has reached critical market dominance or far-reaching standardization efforts may provide similar potentials.

\section{Conclusion \& outlook}

In this paper, we investigated how and by which factors the implementation of blockchain technologies is affected in energy sector use cases. We have constructed an integrated research framework based on factors from DOI, TOE and institutional economics. On this foundation, we conducted semi-structured in-depth interviews with 22 experts from the energy sector, blockchain enterprises and research institutions. Based on the interviews, we used axial coding to identify 
constructs related to the theory and analyzed their impact on the blockchain use cases.

Both grid services and microgrids are clearly affected by power induced factors and technological constraints. Especially permissioned blockchains exhibit properties that equate with these factors, appropriating a system controlled by only few entities. The wholesale electricity trade exhibits strong relationships to technological constraints, while weak links to power-induced and network-related factors can be observed. The effects have counteracting directions, therefore either permissioned or scalable permissionless solutions could fit the requirements of a given project. Both asset management and certification of green electricity are affected by trust-supplementing factors, indicating a system with reliable data and potentially reading access for multiple entities. E-mobility charging infrastructure is strongly affected by network-related factors that indicate a requirement of open and interoperable systems, such a multi-purpose chains.

This is the first scientific study to depict dynamics between blockchain use cases and theory-based determinants. The work provides insight in factors relevant to the implementation of blockchain use cases and the design of economic and political institutions for the regulation of blockchain technologies. However, the study is limited by its focus on the energy sector and is thus lacking generalizability. While we only interviewed about two dozen experts, we do not aim to develop new theories but rather to contribute to the understanding of the dynamics in a specific sociotechnological context. For future research, we aim to supplement our qualitative research with a quantitative analysis to validate the results. We further consider an extension of this approach to other industries.

\section{References}

[1] Baker, J., "The technology-organization-environment framework", In Information systems theory. Springer, 2012, 231-245.

[2] BDEW, "Blockchain in der Energiewirtschaft", 2017.

[3] Bewley, T., "Interviews as a valid empirical tool in economics", The Journal of Socio-Economics 31(4), 2002, pp. 343-353.

[4] Bishop, M., Computer security, Addison-Wesley, Boston, 2008.

[5] Briscoe, B., A. Odlyzko, and B. Tilly, "Metcalfe's law is wrong-communications networks increase in value as they add members-but by how much?", IEEE Spectrum 43(7), 2006, pp. 34-39.

[6] Buchanan, J.M., R.D. Tollison, and G. Tullock, Toward a theory of the rent-seeking society, Texas A \& M Univ Pr, 1980.
[7] Buchman, E., "Tendermint: Byzantine Fault Tolerance in the Age of Blockchains", 2016.

[8] Buis, T., J. Koorn, J. Kroon, L. Kuijt, H. Pruim, and W. Tan, Towards a Decision Model for Blockchain Adoption, 2016.

[9] Burger, C., A. Kuhlmann, P. Richard, and J. Weinmann, "Blockchain in the energy transition. A survey among decision-makers in the German energy industry", dena, 2016.

[10] Castro, M., and B. Liskov, "Practical Byzantine fault tolerance and proactive recovery", ACM Transactions on Computer Systems (TOCS) 20(4), 2002, pp. 398-461.

[11] Coase, R.H., "The nature of the firm", economica 4(16), 1937, pp. 386-405.

[12] Consensys, Grid + - Welcome to the Future of Energy Whitepaper 2.0, 2017.

[13] Corbin, J., and A. Strauss, "Basics of qualitative research", 2008.

[14] Dalkmann, U., “Ansätze im Smart Market für EnergieVertriebsunternehmen”, In Smart Market - Vom Smart Grid zum intelligenten Energiemarkt. Springer Fachmedien Wiesbaden, Wiesbaden, 2014, 319-342.

[15] Davidson, S., P. De Filippi, and J. Potts, "Economics of blockchain", 2016.

[16] Depietro, R., E. Wiarda, and M. Fleischer, "The context for change", In L. Tornatzky, M. Fleischer and A.

Chakrabarti, eds., The processes of technological innovation. Lexington Books, 1990, 151-175.

[17] Flyvbjerg, B., Making social science matter: Why social inquiry fails and how it can succeed again, Cambridge university press, 2001.

[18] Group, H.A.W., Hyperledger Architecture, Volume 1 Introduction to Hyperledger Business Blockchain Design Philosophy and Consensus,

[19] GrünStromJetons, "GrünStromJetons - Press Release", StromDAO, 2016. https://stromdao.de/gruenstromjetons

[20] Hasse, F., A. von Perfall, T. Hillebrand, and E. Smole, "Blockchain - Chance für Energieverbraucher?", pwc, 2016.

[21] innogy, "Share and Charge", 2017. https://shareandcharge.com

[22] International Organization for Standardization, "ISO/TC 307 Blockchain and distributed ledger technologies", 2017. https://www.iso.org/committee/6266604.html

[23] Jae Kwon, and E. Buchman, "Cosmos - A Network of Distributed Ledgers”, Whitepaper, 2017. https://cosmos.network/whitepaper

[24] Kuan, K.K.Y., and P.Y.K. Chau, "A perception-based model for EDI adoption in small businesses using a technology?", Information \& Management 38(8), 2001, pp. 507-521.

[25] Lyytinen, K., and J. Damsgaard, "What's wrong with the diffusion of innovation theory?", In Diffusing software product and process innovations. Springer, 2001, 173-190. 
[26] Mengelkamp, E., J. Gärttner, K. Rock, S. Kessler, L. Orsini, and C. Weinhardt, "Designing microgrid energy markets", Applied Energy, 2017.

[27] Murphy, S., R.D. Smith, and A. de Fazekas, "Unlocking the blockchain: A global legal and regulatory guide", Norton Rose Fulbright, 2016.

[28] Nakamoto, S., Bitcoin: A Peer-to-Peer Electronic Cash System, 2008. https://bitcoin.org/bitcoin.pdf

[29] Patton, M.Q., Qualitative evaluation and research methods, Sage, Newbury Park, 1990.

[30] Pilkington, M., "Blockchain Technology: Principles and Applications", Research Handbook on Digital

Transformations, edited by F. Xavier Olleros and Majlinda Zhegu, 2016.

[31] Ponton, "Enerchain P2P Trading Project - European Energy Trading Firms test peer-to-peer Trading over the Blockchain", 2017.

https://enerchain.ponton.de/index.php/21-enerchain-p2ptrading-project

[32] Poon, J., and V. Buterin, Plasma: Scalable Autonomous Smart Contracts, 2017.

[33] Porter, M.E., Competitive strategy - Techniques for analyzing industries and competitors, Simon and Schuster, ISBN 1416590358, 2008.

[34] Rogers, E.M., Diffusion of innovations, Simon and Schuster, 4th Edition, ISBN 1451602472, 2010.

[35] Schatsky, D., and C. Muraskin, "Beyond bitcoin Blockchain is coming to disrupt your industry", Deloitte University Press, 2015.

[36] Scholtka, B., and J. Martin, "Blockchain-Ein neues Modell für den Strommarkt der Zukunft?", Recht der Energiewirtschaft 17(3), 2017, pp. 113-119.

[37] Shapiro, C., and H.R. Varian, Information rules: a strategic guide to the network economy, Harvard Business Press, 1998.

[38] Shier, D., W. Wu, and A. Pentland, "Blockchain \& Infrastructure (Identity, Data Security)", Massachusetts Institute of Technology - Connection Science 1(3), 2016.

[39] Slock.it, "Private vs. Public Chain", 2017. https://blog.slock.it/public-vs-private-chain-7b7ca45044f

[40] Swan, M., Blockchain: Blueprint for a New Economy, O'Reilly and Associates, 2015.

[41] Tapscott, D., and A. Tapscott, Blockchain Revolution: How the technology behind bitcoin is changing money business, and the world, Penguin Publishing Group, New York, USA, 2016.

[42] Venkatesh, V., and F.D. Davis, "A theoretical extension of the technology acceptance model: Four longitudinal field studies", Management science 46(2), 2000, pp. 186-204.

[43] Venkatesh, V., and X. Zhang, "Unified theory of acceptance and use of technology: US vs. China”, Journal of Global Information Technology Management 13(1), 2010, pp. 5-27.
[44] Voshmgir, S., "Blockchains, Smart Contracts und das Dezentrale Web", 2016. https:/www.technologiestiftungberlin.de/fileadmin/daten/media/publikationen/170130_Bloc kchainStudie.pdf

[45] Vukolić, M., "The quest for scalable blockchain fabric: Proof-of-work vs. BFT replication", International Workshop on Open Problems in Network Security, Springer (2015), $112-125$

[46] Wood, G., "Ethereum: A secure decentralised generalised transaction ledger", Ethereum Project Yellow Paper 151, 2014.

[47] Wood, G., Polkadot, https://github.com/w3f/polkadotwhite-paper/raw/master/PolkaDotPaper.pdf, 2016.

[48] Yin, R.K., Case study research, Sage publications Design and methods, 2013.

[49] Yli-Huumo, J., D. Ko, S. Choi, S. Park, and K. Smolander, "Where Is Current Research on Blockchain Technology?-A Systematic Review”, PloS one 11(10), 2016, pp. e0163477.

[50] Zhu, K., K.L. Kraemer, and J. Dedrick, "Information Technology Payoff in E-Business Environments", Journal of Management Information Systems 21(1), 2014, pp. 17-54.

[51] "Bitcoin and Ethereum vs Visa and PayPal Transactions per second", Altcoin Today. $\mathrm{http}: / / \mathrm{www}$.altcointoday.com/bitcoin-ethereum-vs-visapaypal-transactions-per-second/

[52] "Energy companies join forces with Rocky Mountain Institute and Grid Singularity to launch global blockchain initiative”, Rocky Mountain Institute, 2017.

https://www.rmi.org/about/news-and-press/press-releaseenergy-web-foundation-launch/

[53] "Fees for Selling and Accepting Payments", Paypal, 2017. https://www.paypal.com/us/webapps/mpp/merchantfees

[54] "Conjoule closes Series A investment from innogy Innovation Hub and TEPCO”, Bloomberg, 2017.

http://www.bloomberg.com/research/stocks/news/article.asp? docKey $=600-201707110130 \mathrm{JCN}$

[55] "Gridchain - Blockchain-Based Process Integration for the Smart Grids of the Future", Ponton, 2017. https:/enerchain.ponton.de/index.php/16-gridchainblockchain-based-process-integration-for-the-smart-grids-ofthe-future

[56] "TenneT unlocks distributed flexibility via IBM Blockchain - United States", IBM, 2017. https://www03.ibm.com/press/us/en/pressrelease/52243.wss\#release

[57] "The Promise of Platform-based Grids - Rocky Mountain Institute", Rocky Mountain Institute, 2017. https://rmi.org/news/promise-platform-based-grids/

[58] "How Blockchain Is Helping China Go Greener Hyperledger", Hyperledger, 2017. https://www.hyperledger.org/news/2017/03/20/3-20-17-pcmagazine-how-blockchain-is-helping-china-go-greener 